\title{
Proof of the functional equation for the Riemann zeta-function
}

\author{
Jay Mehta and P. -Y. Zhu
}

\begin{abstract}
In this article, we shall prove a result which enables us to transfer from finite to infinite Euler products. As an example, we give two new proofs of the infinite product for the sine function depending on certain decompositions. We shall then prove some equivalent expressions for the functional equation, i.e. the partial fraction expansion and the integral expression involving the generating function for Bernoulli numbers. The equivalence of the infinite product for the sine functions and the partial fraction expansion for the hyperbolic cotangent function leads to a new proof of the functional equation for the Riemann zeta function.
\end{abstract}

Keywords. Weierstrass product for the sine function, Riemann zeta function, functional equation, partial fraction expansion for the cotangent function

2010 Mathematics Subject Classification. 11M06

\section{Introduction and statement of the result}

Let

$$
\zeta(s)=\sum_{n=1}^{\infty} \frac{1}{n^{s}}
$$

for $\sigma=\operatorname{Re} s>1$ denote the Riemann zeta-function. This is continued analytically to a meromorphic function over the whole plane with a unique simple pole at $s=1$ with residue 1 . The analytic continuation is done by some expressions in $0<\sigma<1$ and by the functional equation

$$
\pi^{-\frac{s}{2}} \Gamma\left(\frac{s}{2}\right) \zeta(s)=\pi^{-\frac{1-s}{2}} \Gamma\left(\frac{1-s}{2}\right) \zeta(1-s)
$$

for $\sigma<0$. But to go into the critical strip, one needs the Euler product. Euler products in general have been one of the most important objects of research from the time of Euler. For instance, the most famous Euler product for the Riemann zeta-function reads

$$
\zeta(s)=\prod_{p}\left(1-p^{-s}\right)^{-1}
$$

for Re $s>1$ and where $p$ runs through all the primes. Recalling the proof, we find that we need to start from a finite product $\prod_{p \leq X}\left(1-p^{-s}\right)^{-1}$ and to prove that the remainder factor is over those integers whose prime factors are $\geq X$, which approaches to 1 as $X \rightarrow \infty$. Thus we can say that we could reach the infinite product from its finite part.

In this article we are mainly concerned with the infinite product for the sine function and prove that it gives a new proof for the functional equation for the Riemann zeta-function (see Theorem 3.2).

In some literature one finds a rather winding proof of the infinite product expression for the sine function (2.9), cf. Remark 2.2 below. To circumvent such complication, we shall use the following handy lemma. 
Lemma 1.1. Suppose $a_{k}$ be such that $\sum_{k=1}^{\infty}\left|a_{k}\right|<\infty$ and a fortiori $\left|a_{k}\right|<\frac{1}{2}$ for $k \geq N$ for some $N \in \mathbb{N}$. Suppose $b_{k}(n)$ is an increasing sequence in $k$ and such that $b_{k}(n)=O\left(n^{-\delta}\right)$ with some $\delta>1$. Then for $m \in \mathbb{N} \cup\{0\}$

$$
\prod_{k=m+1}^{n}\left(1+a_{k}+b_{k}(n)\right) \rightarrow \prod_{k=m+1}^{\infty}\left(1+a_{k}\right)
$$

as $n \rightarrow \infty$.

Proof. We write the left-hand side of (1.4) as

$$
\prod_{k=m+1}^{n}\left(1+a_{k}\right) \Pi_{1}
$$

where $\Pi_{1}=\prod_{k=m+1}^{n}\left(1+\frac{b_{k}(n)}{1+a_{k}}\right)$. We apply the inequality

$$
\prod_{k=m+1}^{n}\left(1+c_{k}\right) \leq \exp \left(\sum_{k=m+1}^{n} c_{k}\right)
$$

to deduce that

$$
\begin{aligned}
\Pi_{1} & =\prod_{k=m+1}^{n}\left(1+\frac{b_{k}(n)}{1+a_{k}}\right) \leq \exp \left(\sum_{k=m+1}^{n} \frac{b_{k}(n)}{1+a_{k}}\right) \\
\leq \exp \left(2 \sum_{k=m+1}^{n} b_{k}(n)\right) & =\exp \left(O\left(\frac{1}{n^{\delta-1}}\right)\right)
\end{aligned}
$$

which goes to 1 as $n \rightarrow \infty$.

On the other hand, note that for $0 \leq x \leq \rho<e^{-1}<1$ we have $1-x \geq e^{-\rho^{-1} x}$. Hence for some $c>0$

$$
\Pi_{1} \geq \prod_{k=m+1}^{n}\left(1-2\left|b_{k}(n)\right|\right) \geq \exp \left(-c \sum_{k=m+1}^{n}\left|b_{k}(n)\right|\right),
$$

which $\rightarrow 1$ as above.

Theorem 1.2. Suppose a polynomial $P(x)=P_{n}(x)$ of degree $n$ (or a linear combination thereof) has a decomposition into the product and after pairing the conjugate roots together, we have

$$
P(x)=P(0) \prod_{k=1}^{\left[\frac{n}{2}\right]}\left(1+a_{k}+b_{k}(n)\right)
$$

where $a_{k}=a_{k}(x)$ and $b_{k}(n)=b_{k}(n, x)$ are subject to conditions in Lemma 1.1 and that $\Pi(x)=$ $\lim _{n \rightarrow \infty} P_{n}(x)$ exists. Then we have the infinite product expression

$$
\Pi(x)=P(0) \prod_{k=1}^{\infty}\left(1+a_{k}\right)
$$




\section{Examples}

It is generally known that the sine function $\sin \theta$ is $\operatorname{simpler}$ in the sense that near $0 \sin \theta$ behaves like $\theta$, which is often used in applied disciplines and we refer to this as the Principle.

We give two examples on the grounds of the Principle. The first is the well-known infinite product expression (2.9) for the sine function and the second is the product expression for the hyperbolic sine function.

The first usually follows either from the Weierstrass product expression for the gamma function and the reciprocity formula (3.31) below or from the partial fraction expansion for the cotangent function, or what amounts to the same, the partial fraction expansion (3.42) for the hyperbolic cotangent function. All of these are known to be equivalent to the functional equation for the Riemann zetafunction. See Theorem 3.2 below.

Corollary 2.1 resp. Corollary 2.3 depends on the decomposition of the Chebyshev polynomial resp. the polynomial $z^{n}-1$.

Corollary 2.1. The decomposition

$$
\sin x=(2 n+1) \sin \frac{x}{2 n+1} \prod_{k=1}^{n}\left(1-\frac{\sin ^{2} \frac{x}{2 n+1}}{\sin ^{2} \frac{k \pi}{2 n+1}}\right),
$$

leads to the infinite product for the sine function

$$
\sin \pi x=\pi x \prod_{k=1}^{\infty}\left(1-\frac{x^{2}}{k^{2}}\right)
$$

which of course leads to the equality of analytic functions by analytic continuation.

Proof. We use well-known Chebyshev polynomials, cf. e.g. [CKK17, pp. 104-107], [CKT09, pp. 10-16] and [MH02]. Here we are concerned with the counterpart (which is not of the first kind) of the Chebyshev polynomial $U_{n}(x)$ of the second kind. A familiar problem in calculus is to find the value of $\sin \frac{\pi}{5}$ since it gives the value of the golden ratio. We may also work with the counterpart $P_{n}(x)$ of $U_{n}(x)$ since $P_{4}\left(\sin \frac{\pi}{5}\right)=0$. Recall the formula [CKT09, (1.36)]:

$$
\sin (2 n+1) \theta=\sin \theta \sum_{k=0}^{n}\left(\begin{array}{l}
2 n+1 \\
2 k+1
\end{array}\right)(-1)^{k}\left(1-\sin ^{2} \theta\right)^{n-k} \cdot \sin ^{2 k} \theta=\sin \theta P_{n}\left(\sin ^{2} \theta\right),
$$

say. The counterpart of this is

$$
\sin (2 n+1) \theta=\sin \theta U_{2 n}(\cos \theta) .
$$

We write $u=\sin ^{2} \theta$. Then correspondingly to [CKT09, Remark 1.1.], $P_{n}(u)=0$ if and only if $(2 n+1) \theta=k \pi,-n \leq k \leq n$ in the interval $\left[-\frac{\pi}{2}, \frac{\pi}{2}\right]$ to which we may apparently restrain the range of $u$. Hence pairing the symmetric ones together, we have

$$
P_{n}(u)=P_{n}(0) \prod_{k=1}^{n}\left(1-\frac{u}{\sin ^{2} \frac{k \pi}{2 n+1}}\right),
$$

with the constant term $P_{n}(0)$ given by $\lim _{\theta \rightarrow 0} \frac{\sin (2 n+1) \theta}{\sin \theta}=2 n+1$ where we used the above principle. Putting $u=\sin ^{2} \frac{x}{2 n+1}$ (i.e. $\left.\sin ^{2} \theta=\sin ^{2} \frac{x}{2 n+1}\right)$

$$
\frac{\sin x}{\sin \frac{x}{2 n+1}}=P_{n}\left(\sin ^{2} \frac{x}{2 n+1}\right)=(2 n+1) \prod_{k=1}^{n}\left(1-\frac{\sin ^{2} \frac{x}{2 n+1}}{\sin ^{2} \frac{k \pi}{2 n+1}}\right),
$$


or $(2.8)$.

Applying Theorem 1.2 with $-\frac{\sin ^{2} \frac{x}{2 n+1}}{\sin ^{2} \frac{k \pi}{2 n+1}}=a_{k}+b_{k}(n)$, where $a_{k}=-\frac{x^{2}}{\pi^{2} k^{2}}, b_{k}(n)=O\left(\frac{1}{n^{2}}\right)$, the right-hand side of (2.8) leads to $x \prod_{k=1}^{n}\left(1-\frac{x^{2}}{\pi^{2} k^{2}}\right)$.

Remark 2.2. In literature one sees a rather technical and ad hoc proof as the following. Using the inequalities

$$
\frac{2}{\pi} x \leq \sin x \leq x
$$

for $\left[-\frac{\pi}{2}, \frac{\pi}{2}\right]$, one finds the estimate

$$
\frac{\sin ^{2} \frac{x}{2 n+1}}{\sin ^{2} \frac{k \pi}{2 n+1}} \geq \frac{x^{2}}{4 k^{2}}
$$

and applies this to the right-hand side of

$$
\frac{\sin x}{(2 n+1) \sin \frac{x}{2 n+1} \prod_{k=1}^{m}\left(1-\frac{\sin ^{2} \frac{x}{2 n+1}}{\sin ^{2} \frac{k \pi}{2 n+1}}\right)}=\prod_{k=m+1}^{n}\left(1-\frac{\sin ^{2} \frac{x}{2 n+1}}{\sin ^{2} \frac{k \pi}{2 n+1}}\right) .
$$

Then one concludes that the truncated product approaches to 1 as $n \rightarrow \infty$. Then putting it back and taking the limit as $m \rightarrow \infty$ to conclude (2.9).

Now we turn to the second corollary in which we shall prove the infinite product expression for the hyperbolic sine function.

Corollary 2.3. The decomposition (2.21) amounts to (2.27), which as $n \rightarrow \infty$ leads to the product expression for $\sinh x$ :

$$
\sinh x=\prod_{k=1}^{\infty}\left(1+\frac{x^{2}}{\pi^{2} k^{2}}\right) .
$$

By the change of variable $x \leftrightarrow i \pi x$, we arrive at (2.9).

Proof. Let $1<n \in \mathbb{N}$ be an odd integer. It is clear that we have the decomposition

$$
z^{n}-1=(z-1) \prod_{k=1}^{n-1}\left(z-\exp \left(2 \pi i \frac{k}{n}\right)\right)
$$

which amounts to

$$
z^{n}-1=(z-1) \prod_{k=1}^{\frac{n-1}{2}}\left(z^{2}-\left(\cos 2 \pi \frac{k}{n}\right) 2 z+1\right),
$$

on pairing the conjugates. Putting $z=\frac{x}{y}$, we find that

$$
\left.x^{n}-y^{n}=(x-y) \prod_{k=1}^{\frac{n-1}{2}}\left(x^{2}-\left(\cos 2 \pi \frac{k}{n}\right) 2 x y+y^{2}\right)\right) .
$$

Here we make a clever choice

$$
x=1+\frac{x}{n}, \quad y=1-\frac{x}{n} .
$$

Then (2.19) reads

$$
Q_{n}(x):=\left(1+\frac{x}{n}\right)^{n}-\left(1-\frac{x}{n}\right)^{n}=\frac{2 x}{n} \prod_{k=1}^{\frac{n-1}{2}} 2^{2} A_{k}(x),
$$


where

$$
\begin{aligned}
A_{k}(x) & =\frac{1-\cos \frac{2 \pi k}{n}}{2}+\frac{1+\cos \frac{2 \pi k}{n}}{2} \frac{x^{2}}{n^{2}}=\sin ^{2} \frac{\pi k}{n}+\left(\cos ^{2} \frac{\pi k}{n}\right) \frac{x^{2}}{n^{2}} \\
& =\sin ^{2} \frac{\pi k}{n}\left(1+\left(\cot ^{2} \frac{\pi k}{n}\right) \frac{x^{2}}{n^{2}}\right) .
\end{aligned}
$$

We appeal to

$$
\cot x=\frac{1}{x}+O(x), \quad x \rightarrow 0
$$

so that one of the second factor in the last equality in (2.21) is

$$
1+\left(\cot ^{2} \frac{\pi k}{n}\right) \frac{x^{2}}{n^{2}}=1+\frac{x^{2}}{\pi^{2} k^{2}}+O\left(\left(\frac{x}{n}\right)^{2}\right)
$$

Hence (2.22) becomes

$$
A_{k}(x)=\sin ^{2} \frac{\pi k}{n}\left(1+a_{k}+b_{k}(n)\right)
$$

where $a_{k}=\frac{x^{2}}{\pi^{2} k^{2}}$. Substituting this in $(2.21)$ we conclude that

$$
Q_{n}(x)=\frac{2^{n} x}{n} \prod_{k=1}^{n-1} \sin \frac{\pi k}{n} \prod_{k=1}^{\frac{n-1}{2}}\left(1+a_{k}+b_{k}(n)\right) .
$$

The first product of sine values is well-known, e.g. [CKK17, (106),p 231] in the form

$$
\prod_{k=1}^{n-1} \sin \frac{\pi k}{n}=\frac{n}{2^{n-1}}
$$

Hence (2.25) amounts to

$$
\left(1+\frac{x}{n}\right)^{n}-\left(1-\frac{x}{n}\right)^{n}=Q_{n}(x)=2 x \prod_{k=1}^{\frac{n-1}{2}}\left(1+a_{k}+b_{k}(n)\right)
$$

Remark 2.4. Example 2.3 seems more intrinsic to the product expansion (2.9), including the cotangent function. Note that in the proof we used (2.23), which is a consequence of the Principle.

\section{Functional equation for the Riemann zeta-function}

For details on the functional equation for the Riemann zeta-function, there is enormous amount of literature. We mainly refer to [KT15], [Ko54] and [Ti86].

Lemma 3.1. We have the Mellin transform formulas for $\sigma>0$

$$
\begin{gathered}
e^{-x}=\frac{1}{2 \pi i} \int_{-\infty}^{\infty} \Gamma(s) x^{-s} \mathrm{~d} x \\
\int_{0}^{\infty} \frac{1}{1+x^{2}} x^{s-1} \mathrm{~d} x=\frac{\pi}{2 \sin \frac{\pi}{2} s},
\end{gathered}
$$


the duplication formula

$$
\Gamma(s)=\sqrt{\pi}^{-1} 2^{s-1} \Gamma\left(\frac{s}{2}\right) \Gamma\left(\frac{s+1}{2}\right)
$$

the reciprocal relation

$$
\Gamma(s) \Gamma(1-s)=\frac{\pi}{\sin \pi s}
$$

and the Stirling formula: for $t \geq \delta<0$ uniformly in $\sigma_{1} \leq \sigma \leq \sigma_{2}$.

$$
|\Gamma(s)|=\sqrt{2 \pi} \exp \left(-\frac{\pi}{2}|t|\right)|t|^{\sigma-\frac{1}{2}}\left(1+O\left(\frac{1}{|t|}\right)\right) .
$$

The partial fraction expansion for the hyperbolic cotangent function may be stated as

$$
\begin{aligned}
\frac{1}{e^{x}-1} & =\frac{1}{x}-\frac{1}{2}+\frac{x}{2 \pi^{2}} \sum_{n=1}^{\infty} \frac{1}{\left(\frac{x}{2 \pi}\right)^{2}+n^{2}} \\
& =\frac{1}{x}-\frac{1}{2}+\frac{1}{2 \pi^{2}} \sum_{n=1}^{\infty} \frac{1}{n^{2}} \frac{x}{\left(\frac{x}{2 \pi n}\right)^{2}+1}
\end{aligned}
$$

where the series is absolutely convergent. See (3.42) below.

On the other hand, the Mellin transform formula

$$
\Gamma(s) \zeta(s)=\int_{0}^{\infty} \frac{1}{e^{x}-1} x^{s-1} \mathrm{~d} x
$$

is well-known which is valid for $\sigma>1$. By the Mellin inversion formula we have

$$
\frac{1}{e^{x}-1}=\frac{1}{2 \pi i} \int_{(c)} \Gamma(s) \zeta(s) x^{-s} \mathrm{~d} s
$$

valid for $c>1$, where we mean by $(c)$ the vertical integration path $\sigma=c,-\infty<t<\infty$ and also subsequently.

By the Stirling formula (and the polynomial growth of the zeta-function in $t$ ), we may shift the line in (3.35) of integration to $\sigma=d,-1<d<0$. In doing so we pass the simple poles of $\zeta(s)$ and $\Gamma(s)$ at $s=1$ and $s=0$, respectively. The residues are $\frac{1}{x}$ and $\zeta(0)=-\frac{1}{2}$. Hence (3.35) leads to

$$
\frac{1}{e^{x}-1}-\frac{1}{x}+\frac{1}{2}=\frac{1}{2 \pi i} \int_{(d)} \Gamma(s) \zeta(s) x^{-s} \mathrm{~d} s .
$$

By the Mellin inversion, this implies

$$
\Gamma(s) \zeta(s)=\int_{0}^{\infty}\left(\frac{1}{e^{x}-1}-\frac{1}{x}+\frac{1}{2}\right) x^{s-1} \mathrm{~d} x
$$

which is valid for $-1<\sigma<0$. Noting that the integrand is the series part of (3.33) which is absolutely convergent, we may integrate it term by term. We then arrive at

$$
\Gamma(s) \zeta(s)=\frac{1}{2 \pi^{2}} \sum_{n=1}^{\infty} \frac{1}{n^{2}} \int_{0}^{\infty} \frac{1}{\left(\frac{x}{2 \pi n}\right)^{2}+1} x^{s-1} \mathrm{~d} x .
$$

By the change of variable $x=2 \pi n y$, the integral in (3.38) becomes

$$
(2 \pi n)^{s+1} \int_{0}^{\infty} \frac{1}{1+y^{2}} y^{s+1-1} \mathrm{~d} x=(2 \pi n)^{s+1} \frac{\pi}{2 \sin \frac{\pi}{2}(s+1)}
$$


by (3.29). Hence (3.38) amounts to

$$
\begin{aligned}
& \Gamma(s) \zeta(s)=\frac{1}{2 \pi^{2}} \sum_{n=1}^{\infty} \frac{1}{n^{2}}(2 \pi n)^{s+1} \frac{\pi}{2 \sin \frac{\pi}{2}(s+1)} \\
& =(2 \pi)^{s-1} \frac{\pi}{2 \sin \frac{\pi}{2}(s+1)} \sum_{n=1}^{\infty} \frac{1}{n^{1-s}} .
\end{aligned}
$$

The resulting series is absolutely convergent for $\sigma<0$ and indeed is $\zeta(1-s)$. Now dividing both sides of (3.40) by $\Gamma\left(\frac{s+1}{2}\right)$ and using (3.30), we transform it into

$$
\begin{aligned}
& \frac{1}{\sqrt{\pi}} 2^{s-1} \Gamma\left(\frac{s}{2}\right) \zeta(s)=\frac{1}{2 \pi^{2}} \sum_{n=1}^{\infty} \frac{1}{n^{2}}(2 \pi n)^{s+1} \frac{\pi}{2 \sin \frac{\pi}{2}(s+1) \Gamma\left(\frac{s+1}{2}\right)} \\
& =(2 \pi)^{s-1} \frac{\Gamma\left(1-\frac{s+1}{2}\right)}{2} \zeta(1-s)
\end{aligned}
$$

by (3.31). This leads to the functional equation (1.2) and proves the necessity part of the following Theorem 3.2 (ii).

Theorem 3.2. $\quad$ (i) The infinite product expansion for the sine function and the partial fraction expansion for the cotangent function are equivalent.

(ii) The partial fraction expansion for the cotangent function and the functional equation (1.2) for the Riemann zeta-function are equivalent.

(iii) Theorem 1.2 implies the infinite product expansion for the sine function and a fortiori, the functional equation (1.2) for the Riemann zeta-function.

It remains to prove sufficiency part in (ii) that the functional equation (1.2) for the Riemann zeta-function implies the partial fraction expansion for the cotangent function. Although this is done in [Ko54] for the case of the rational and quadratic fields, we give a proof.

Lemma 3.3. The functional equation (1.2) implies the partial fraction expansion for the hyperbolic cotangent function (3.33) in the form

$$
\frac{x}{e^{x}-1}=1-\frac{x}{2}+\frac{x^{2}}{2 \pi^{2}} \sum_{n=1}^{\infty} \frac{1}{\left(\frac{x}{2 \pi}\right)^{2}+n^{2}}
$$

for $x>0$ or for $\operatorname{Re} x>0$

Proof. We show that the function

$$
L(x):=\sqrt{\pi} \sum_{n=1}^{\infty} e^{-2 \pi n x}=\frac{1}{2 \pi i} \int_{(c)} \frac{\pi^{\frac{1}{2}-s}}{2 \cos \frac{\pi}{2} s} \frac{\Gamma\left(\frac{s}{2}\right)}{\Gamma\left(\frac{1-s}{2}\right)} \zeta(s) x^{-s} \mathrm{~d} s, \quad c>1
$$

gives the partial fraction expansion. First, we need to prove (3.43). By (3.30), the integrand becomes $\Gamma(s)(2 \pi x)^{-s}$. Hence by (3.28), the integral in (3.43) is $\sqrt{\pi} e^{-2 \pi x}$, proving (3.43).

Form here, the procedure is similar to that used in proving the necessity part. Although the Stirling formula (3.32) does not give rapid increase, we have the cosine function in the denominator and it gives the same effect as the Stirling formula. Hence shifting the integration path to $\sigma=d$, $-1<d<0$, we pass two poles at $s=0,1$ with residues $1,-\frac{x}{2}$. Hence

$$
L(x)=1-\frac{x}{2}+\frac{1}{2 \pi i} \int_{(d)} \frac{\pi^{\frac{1}{2}-s}}{2 \cos \frac{\pi}{2} s} \frac{\Gamma\left(\frac{s}{2}\right)}{\Gamma\left(\frac{1-s}{2}\right)} \zeta(s) x^{-s} \mathrm{~d} s .
$$


Using the functional equation (1.2), the integrand of the integral in (3.44) becomes

$$
\frac{\pi^{\frac{1}{2}-s}}{2 \cos \frac{\pi}{2} s} \Gamma\left(\frac{s}{2}\right) \zeta(s)=\frac{1}{2 \cos \frac{\pi}{2} s} \zeta(1-s) .
$$

But $\zeta(1-s)$ can be expanded into Dirichlet series $\sum_{n=1}^{\infty} n^{s-1}$, so that altogether, by making the change of variable $s \leftrightarrow 1-s$

$$
\begin{aligned}
L(x) & =1-\frac{x}{2}+\frac{x}{2 \pi^{2}} \sum_{n=1}^{\infty} \frac{1}{2 \pi i} \int_{(1-d)} \frac{1}{2 \cos \frac{\pi}{2} s}\left(\frac{2 \pi n}{x}\right)^{-s} \mathrm{~d} s \\
& =1-\frac{x}{2}+\frac{x}{2 \pi^{2}} \sum_{n=1}^{\infty} \frac{1}{1+\left(\frac{2 \pi n}{x}\right)^{2}} .
\end{aligned}
$$

by (3.29), completing the proof.

Remark 3.4. In the discussion in [KT07, §5.4, pp. 97-102], the Weierstrass product expression for the sine function has an auxiliary role and shows up through the reciprocal relation for the gamma function. Our discussion has made clear that the Weierstrass product expression for the sine function directly leads to the functional equation and that for the gamma function not. This is because the expression for the sine function is complete in itself, i.e. it holds without convergence factor while that for the gamma function needs a convergence factor. This is why the gamma function has been treated as an auxiliary factor in the completed zeta-function in some literature.

It is possible to treat the integral in (3.34) as a deformed Hankel contour and obtain the universal expression for $\Gamma(s) \zeta(s)$ and proceed to the deduction of the functional equation.

A proof of the functional equation in its asymmetric form is given in [Ge66, pp. 67-71]. The method starts from the formula (3.36) and uses the contour deformation.

Acknowledgment. The authors would like to thank Prof. Shigeru Kanemitsu for his guidance and encouragement. The authors would also like to thank the referee(s) for the thorough perusal of the paper.

\section{References}

[CKK17] K. Chakraborty, S. Kanemitsu and T. Kuzumaki, A quick introduction to complex analysis, World Sci., Singapore etc. 2017.

[CKT09] K. Chakraborty, S. Kanemitsu and H. Tsukada, Vistas of special functions II, World Sci., New Jersey-LondonSingapore etc. 2009.

[Ge66] A.O. Gel'fond, Residues and their applications, Nauka, Moscow 1966.

[KT07] S. Kanemitsu and H. Tsukada, Vistas of special functions, World Scientific, Singapore etc. 2007.

[KT15] S. Kanemitsu and H. Tsukada, Contributions to the theory of zeta-functions: the modular relation supremacy, World Sci., Singapore etc. 2015.

[Ko54] N.S. Koshlyakov, Investigation of some questions of analytic theory of the rational and quadratic fields, I (Russian), Izv. Akad. Nauk SSSR, Ser. Mat. 18 (1954), 113-144, 213-260, 307-326, Errata: ibid. 19 (1955), 271 (in Russian).

[MH02] J.C. Mason and D.C. Handscomb, Chebyshev polynomials, Taylor \& Francis, New York 2002.

[Na66] I.P. Natanson, Constructive function theory. Vol. I-III, Frederick Ungar Publ. Co., New York 1966.

[Ti86] E.C. Titchmarsh, The Theory of the Riemann Zeta-Function, (second edition revised by D. R. Heath-Brown), OUP, Oxford 1986. 


\section{Jay Mehta}

Department of Mathematics

Sardar Patel University

Vallabh Vidyanagar 388120

Gujarat, India

e-mail: jay_mehta@spuvvn.edu

\section{Pengyu Zhu}

Shandong University, Taishan School

Shanda Nanlu No. 27, Jinan-250110

People's Republic of China.

e-mail: zhupengyu1002@qq.com 